\title{
A Modified Predictor-Corrector Formula For Solving Ordinary Differential Equation Of First Order And First Degree
}

\author{
Mahtab Uddin And M. A. Ullah* \\ Department of Mathematics, University of Chittagong, Chittagong-4331, Bangladesh.
}

\begin{abstract}
We are proposing a modified form of the Milne's Predictor-Corrector formula for solving ordinary differential equation of first order and first degree. Here we are approximating the value of the dependent variable under five initial conditions (where Milne takes four initial conditions) and then improving this value (closer to the exact value) by proper substitution in the formulae. This process is an iterative way to obtain the values and the process continuing until we get a proper level of accuracy.
\end{abstract}

Keywords: ODE, Milne's modified Predictor-Corrector, quantitative comparison, accuracy

\section{Introduction}

In the methods so far described to solve an ordinary differential equation over an interval, only the value of $y$ at the beginning of the interval was required. Now in the Predictor-Corrector methods, four prior values are needed for finding the value of $\mathrm{y}$ at given value of $\mathrm{x}[2,4]$. These methods though slightly complex, have the advantage of giving an estimate of error from successive approximations of $y_{i}$, with $x_{i+1}=x_{i}+h$.

Then from Euler's formula, we have

$$
y_{i+1}=y_{i}+h f\left(x_{i j}, y_{i}\right)
$$

Also from modified Euler's formula [8,9], we have

$$
y_{i+1}=y_{i}+\frac{h}{2}\left[f\left(x_{i}, y_{i}\right)+f\left(x_{i+1}, y_{i+1}\right)\right]
$$

The value of $y_{i+1}$ is first estimate by (1) and then by using (2) gets a better approximation of $y_{i+1}$. This value of $y_{i+1}$ is again substituted in (2) to find still a better approximation of $y_{i+1}$. This procedure will repeat until two consecutive iterated values of $y_{i+1}$ agree.

This technique of refining an initial crude estimation of $y_{i+1}$ by means of a more accurate formula is known as Predictor-Corrector method [1]. The equation (1) is taken as the predictor, while (2) serves as a corrector of $y_{i+1}$.

To solve the differential equation $y^{f}=f(x, y)$ by this method, first we are to approximate the value of $y_{i+1}$ by predictor formula at $x=x_{n+1}$, then improve this values of $y_{n+1}$ by using the corrector formula after proper substitution. These formulae will be derived from the Newton's formula of forward interpolation $[5,6]$.

\section{Derivation Of Milne's (Modified) Predictor Formula}

We know that Newton's formula of forward interpolation in terms of $y^{y}$ and u is given by

$$
\begin{aligned}
y^{y}=y_{0}+u \Delta & y_{0} \\
& +\frac{u(u-1)}{2 !} \Delta^{2} y_{0}+\frac{u(u-1)(u-2)}{3 !} \Delta^{3} y_{0} \\
& +\frac{u(u-1)(u-2)(u-3)}{4 !} \Delta^{4} y_{0}^{y}+\frac{u(u-1)(u-2)(u-3)(u-4)}{5 !} \Delta^{5} y_{0} \\
& +\cdots \cdots \cdots
\end{aligned}
$$

*Author to whom all correspondences should be made (e-mail: aman@cu.ac.bd). 


$$
\begin{gathered}
=y_{0}+u \Delta y_{0}+\frac{\left(u^{2}-u\right)}{2} \Delta^{2} y_{0}^{4}+\frac{\left(u^{3}-3 u^{2}+2 u\right)}{6} \Delta^{3} y_{0}^{4}+\frac{\left(u^{4}-6 u^{3}+11 u^{2}-6 u\right)}{24} \Delta^{4} y_{0} \\
+\frac{\left(u^{5}-10 u^{4}+35 u^{3}-50 u^{2}+24 u\right)}{120} \Delta^{5} y_{0}^{4}+\cdots \cdots \cdots
\end{gathered}
$$

Where

$$
\begin{array}{r}
u=\frac{x-x_{0}}{h} \\
\text { or, } x=x_{0}+u h
\end{array}
$$

So that $d x=h d u$

Now, integrating (3) over the interval $x_{0}$ to $x_{0}+5$ h. i.e., $u=0$ to $u=5$, we obtain

$$
\begin{aligned}
& \int_{x_{0}}^{x_{0}+5 h} y^{l} d x=h \int_{0}^{5}\left[y_{0}+u \Delta y_{0}+\frac{\left(u^{2}-u\right)}{2} \Delta^{2} y_{0}^{y}+\frac{\left(u^{3}-3 u^{2}+2 u\right)}{6} \Delta^{3} y_{0}\right. \\
& +\frac{\left(u^{4}-6 u^{3}+11 u^{2}-6 u\right)}{24} \Delta^{4} y_{0}^{8} \\
& \left.+\frac{\left(u^{5}-10 u^{4}+35 u^{3}-50 u^{2}+24 u\right)}{120} \Delta^{5} y_{0}+\cdots \cdots \cdots\right] d u \\
& \text { or }_{s}[y]_{x_{0}}^{x_{0}+5 h}=h\left[u y_{0}+\frac{u^{2}}{2} \Delta y_{0}+\frac{1}{2}\left(\frac{u^{3}}{3}-\frac{u^{2}}{2}\right) \Delta^{2} y_{0}^{4}+\frac{1}{6}\left(\frac{u^{4}}{4}-u^{3}+u^{2}\right) \Delta^{3} y_{0}\right. \\
& +\frac{1}{24}\left(\frac{u^{5}}{5}-\frac{3 u^{4}}{2}+\frac{11 u^{3}}{3}-3 u^{2}\right) \Delta^{4} y_{0} \\
& \left.+\frac{1}{120}\left(\frac{u^{6}}{6}-2 u^{5}+\frac{35 u^{4}}{4}-\frac{50 u^{3}}{3}+12 u^{2}\right) \Delta^{5} y_{0}^{4}+\cdots \cdots \cdots\right]_{\mathrm{u}=0}^{\mathrm{w}=5} \\
& \text { or, } y\left(x_{0}+5 h\right)-y\left(x_{0}\right) \\
& =h\left[5 y_{0}+\frac{25}{2} \Delta y_{0}+\frac{1}{2}\left(\frac{125}{3}-\frac{25}{2}\right) \Delta^{2} y_{0}+\frac{1}{6}\left(\frac{625}{4}-125+25\right) \Delta^{3} y_{0}\right. \\
& +\frac{1}{24}\left(625-\frac{1875}{2}+\frac{1375}{3}-75\right) \Delta^{4} y_{0} \\
& \left.+\frac{1}{120}\left(\frac{15625}{6}-6250+\frac{21875}{4}-\frac{6250}{3}+300\right) \Delta^{5} y_{0}^{4}+\cdots \cdots \cdots\right] \\
& \text { or } y_{5}-y_{0}=h\left[5 y_{0}+\frac{25}{2} \Delta y_{0}^{4}+\frac{175}{12} \Delta^{2} y_{0}^{y}+\frac{75}{8} \Delta^{3} y_{0}+\frac{425}{144} \Delta^{4} y_{0}^{y}+\frac{95}{288} \Delta^{5} y_{0}\right. \\
& +\cdots \cdots]
\end{aligned}
$$

Neglecting the terms containing $\Delta^{6}$ and higher orders and substituting $\Delta \equiv E-1$, from (4) we get

$$
\begin{aligned}
y_{5}-y_{0}=h\left[5 y_{0}\right. & +\frac{25}{2}(E-1) y_{0}+\frac{175}{12}(E-1)^{2} y_{0}^{8}+\frac{75}{8}(E-1)^{3} y_{0}+\frac{425}{144}(E-1)^{4} y_{0} \\
+ & \left.\frac{95}{288} \Delta^{5} y_{0}\right]
\end{aligned}
$$




$$
\begin{aligned}
& \text { or } y_{5}=y_{0}+h\left[5 y_{0}+\frac{25}{2}(E-1) y_{0}+\frac{175}{12}\left(E^{2}-2 E+1\right) y_{0}\right. \\
& +\frac{75}{8}\left(E^{3}-3 E^{2}+3 E-1\right) y_{0}+\frac{425}{144}\left(E^{4}-4 E^{3}+6 E^{2}-4 E+1\right) y_{0} \\
& \left.+\frac{95}{288} \Delta^{5} y_{0}\right] \\
& =y_{0}+h\left[\left(5-\frac{25}{2}+\frac{175}{12}-\frac{75}{8}+\frac{425}{144}\right) y_{0}+\left(\frac{25}{2}-\frac{175}{6}+\frac{225}{8}-\frac{425}{36}\right) E y_{0}\right. \\
& \left.+\left(\frac{175}{12}-\frac{225}{8}+\frac{425}{24}\right) E^{2} y_{0}^{4}+\left(\frac{75}{8}-\frac{425}{36}\right) E^{3} y_{0}+\frac{425}{144} E^{4} y_{0}^{4}+\frac{95}{288} \Delta^{5} y_{0}\right] \\
& =y_{0}+h\left[\frac{95}{144} y_{0}-\frac{25}{72} E y_{0}+\frac{25}{6} E^{2} y_{0}-\frac{175}{72} E^{3} y_{0}+\frac{425}{144} E^{4} y_{0}+\frac{95}{288} \Delta^{5} y_{0}\right] \\
& =y_{0}+\frac{5 h}{144}\left[19 y_{0}-10 y_{1}+120 y_{2}-70 y_{3}+85 y_{4}\right]+\frac{95}{288} h \Delta^{5} y_{0}
\end{aligned}
$$

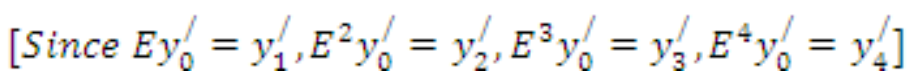

i.e. $y_{5}=y_{0}+\frac{5 h}{144}\left[19 y_{0}-10 y_{1}+120 y_{2}-70 y_{3}+85 y_{4}\right]+\frac{95}{288} h \Delta^{5} y_{0}$

which is required Milne's (modified) predictor formula.

\section{Derivation Of Milne's (Modified) Corrector Formula}

To obtain the corrector formula, we integrate (3) over the interval $x_{0}$ to $x_{0}+4 h$. i.e., $u=0$ to $u=4$ then we get

$$
\begin{aligned}
\int_{x_{0}}^{x_{0}+4 h} y^{4} d x= & h \int_{0}^{4}\left[y_{0}^{4}+u \Delta y_{0}^{4}+\frac{\left(u^{2}-u\right)}{2} \Delta^{2} y_{0}^{y}+\frac{\left(u^{3}-3 u^{2}+2 u\right)}{6} \Delta^{3} y_{0}\right. \\
& +\frac{\left(u^{4}-6 u^{3}+11 u^{2}-6 u\right)}{24} \Delta^{4} y_{0} \\
& \left.+\frac{\left(u^{5}-10 u^{4}+35 u^{3}-50 u^{2}+24 u\right)}{120} \Delta^{5} y_{0}+\cdots \cdots \cdots\right] d u
\end{aligned}
$$$$
\text { or }_{s}[y]_{x_{0}}^{x_{0}+4 h}=h\left[u y_{0}+\frac{u^{2}}{2} \Delta y_{0}+\frac{1}{2}\left(\frac{u^{3}}{3}-\frac{u^{2}}{2}\right) \Delta^{2} y_{0}+\frac{1}{6}\left(\frac{u^{4}}{4}-u^{3}+u^{2}\right) \Delta^{3} y_{0}\right.
$$$$
+\frac{1}{24}\left(\frac{u^{5}}{5}-\frac{3 u^{4}}{2}+\frac{11 u^{3}}{3}-3 u^{2}\right) \Delta^{4} y_{0}
$$$$
\left.+\frac{1}{120}\left(\frac{u^{6}}{6}-2 u^{5}+\frac{35 u^{4}}{4}-\frac{50 u^{3}}{3}+12 u^{2}\right) \Delta^{5} y_{0}^{4}+\cdots \cdots \cdots\right]_{u=0}^{u=4}
$$

$$
\begin{aligned}
\text { or } y\left(x_{0}+4 h\right) & -y\left(x_{0}\right) \\
= & h\left[4 y_{0}^{y}+8 \Delta y_{0}^{y}+\frac{1}{2}\left(\frac{64}{3}-8\right) \Delta^{2} y_{0}^{y}+\frac{1}{6}(64-64+16) \Delta^{3} y_{0}^{y}\right. \\
& +\frac{1}{24}\left(\frac{1024}{5}-384+\frac{704}{3}-48\right) \Delta^{4} y_{0}^{y} \\
& \left.+\frac{1}{120}\left(\frac{2048}{3}-2048+2240-\frac{3200}{3}+192\right) \Delta^{5} y_{0}+\cdots \cdots \cdots\right]
\end{aligned}
$$




$$
\begin{aligned}
\text { or } y_{4}-y_{0}= & h\left[4 y_{0}^{4}+8 \Delta y_{0}^{4}+\frac{20}{3} \Delta^{2} y_{0}^{4}+\frac{8}{3} \Delta^{3} y_{0}^{4}+\frac{14}{45} \Delta^{4} y_{0}^{4}+(0) \Delta^{5} y_{0}\right. \\
& +\cdots \cdots]
\end{aligned}
$$

Neglecting the terms containing $\Delta^{5}$ and higher orders and substituting $\Delta \equiv E-1$, from (6) we get

$$
\begin{aligned}
& y_{4}-y_{0}=h\left[4 y_{0}+8(E-1) y_{0}+\frac{20}{3}(E-1)^{2} y_{0}+\frac{8}{3}(E-1)^{3} y_{0}+\frac{14}{45}(E-1)^{4} y_{0}\right. \\
& \left.+(0) \Delta^{5} y_{0}\right] \\
& \text { or } y_{4}=y_{0}+h\left[4 y_{0}+8(E-1) y_{0}+\frac{20}{3}\left(E^{2}-2 E+1\right) y_{0}+\frac{8}{3}\left(E^{3}-3 E^{2}+3 E-1\right) y_{0}\right. \\
& \left.+\frac{14}{45}\left(E^{4}-4 E^{3}+6 E^{2}-4 E+1\right) y_{0}+(0) \Delta^{5} y_{0}\right] \\
& =y_{0}+h\left[\left(4-8+\frac{20}{3}-\frac{8}{3}+\frac{14}{45}\right) y_{0}+\left(8-\frac{40}{3}+8-\frac{56}{45}\right) E y_{0}\right. \\
& \left.+\left(\frac{20}{3}-8+\frac{28}{15}\right) E^{2} y_{0}^{8}+\left(\frac{8}{3}-\frac{56}{45}\right) E^{3} y_{0}^{8}+\frac{14}{45} E^{4} y_{0}^{4}+(0) \Delta^{5} y_{0}\right] \\
& =y_{0}+h\left[\frac{14}{45} y_{0}^{4}+\frac{64}{45} E y_{0}+\frac{24}{45} E^{2} y_{0}^{4}+\frac{64}{45} E^{3} y_{0}^{4}+\frac{14}{45} E^{4} y_{0}+(0) \Delta^{5} y_{0}\right] \\
& =y_{0}+\frac{2 h}{45}\left[7 y_{0}+32 y_{1}+12 y_{2}+32 y_{3}+7 y_{4}\right]+(0) h \Delta^{5} y_{0}
\end{aligned}
$$

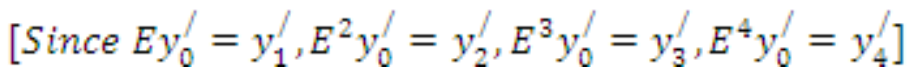

$$
i \cdot e \cdot y_{4}=y_{0}+\frac{2 h}{45}\left[7 y_{0}+32 y_{1}+12 y_{2}+32 y_{3}+7 y_{4}\right]+(0) h \Delta^{5} y_{0}
$$

which is required Milne's (modified) corrector formula.

\section{Generalization Of Milne's (Modified) Predictor-Corrector Formula}

We can write the general form [7] of Milne's (modified) predictor and corrector formulae according to (5) and (7) as follows

$$
\begin{aligned}
& y_{n+1}^{p}=y_{n-4}+\frac{5 h}{144}\left[19 y_{n-4}^{\prime}-10 y_{n-3}^{\prime}+120 y_{n-2}^{/}-70 y_{n-1}^{\prime}+85 y_{n}^{/}\right] \\
& y_{n+1}^{c}=y_{n-3}+\frac{2 h}{45}\left[7 y_{n-3}^{/}+32 y_{n-2}^{/}+12 y_{n-1}^{/}+32 y_{n}^{/}+7 y_{n+1}^{\prime}\right]
\end{aligned}
$$

Here the index p \& c indicates the predicted and corrected values of y respectively.

\subsection{EXAMPLES}

\section{Problem-1:}

Solve $y^{y}=\frac{d y}{d x}=\frac{x+y}{2}$ at $x=2.5$

Where initial values [2] are $\mathrm{y}(0.0)=2.00000000, \mathrm{y}(0.5)=2.63610167, \mathrm{y}(1.0)=3.59488508, \mathrm{y}(1.5)=$ $4.96800007, \mathrm{y}(2.0)=6.87312731$

The analytical solution is $y=4 e^{\frac{a}{2}}-x-2$ 
Problem-2:

Solve $y^{f}=\frac{d y}{d x}=2 e^{x}-y$ at $x=0.5$

Where initial values $[2,5]$ are $\mathrm{y}(0.0)=2.00000000, \mathrm{y}(0.1)=2.01000834, \mathrm{y}(0.2)=2.04013351, \mathrm{y}(0.3)=$ $2.09067703, \mathrm{y}(0.4)=2.16214474$

The analytical solution is $y=e^{x}+e^{-x}$

Problem-3:

Solve $x^{2} y^{y}+x y=1$ at $x=1.5$

Where initial values [5] are $\mathrm{y}(1.0)=1.00000000, \mathrm{y}(1.1)=0.99573653, \mathrm{y}(1.2)=0.98526796, \mathrm{y}(1.3)=$ $0.97104943, \mathrm{y}(1.4)=0.95462303$

The analytical solution is $\quad y=\frac{(\ln x+1)}{x}$

Problem-04:

Solve $y^{y}=\frac{d y}{d x}=3 e^{x}+2 y$ at $x=0.5$

Where initial values $[2,5]$ are $\mathrm{y}(0.0)=0.00000000, \mathrm{y}(0.1)=0.34869552, \mathrm{y}(0.2)=0.81126582, \mathrm{y}(0.3)=$ $1.41677998, \mathrm{y}(0.4)=2.20114869$

The analytical solution is $y=3\left(e^{2 x}-e^{x}\right)$.

Problem-5:

Solve $y^{y}=\frac{d y}{d x}=e^{x-y}+x^{2} e^{-y}$ at $x=0.5$

Where initial values [3] are $\mathrm{y}(0.0)=0.00000000, \mathrm{y}(0.1)=0.10030157, \mathrm{y}(0.2)=0.20218090, \mathrm{y}(0.3)=$ $0.30664524, \mathrm{y}(0.4)=0.41419888$

The analytical solution is $y=\ln \left(e^{x}+\frac{1}{3} x^{3}\right)$

V. Quantitative Comparison Of Numerical Results

\begin{tabular}{|c|c|c|c|c|}
\hline $\begin{array}{l}\text { Problem } \\
\text { No. }\end{array}$ & Exact value & In Milne's method. & $\begin{array}{l}\text { In Milne's (modified) } \\
\text { method. }\end{array}$ & $\begin{array}{l}\text { In Adam-Moulton's } \\
\text { method. }\end{array}$ \\
\hline 01 & 9.46137183 & $\begin{array}{c}9.46150093 \\
7^{\text {th }} \text { iteration } \\
\left|E_{R}\right|=0.00137 \%\end{array}$ & $\begin{array}{c}9.46137662 \\
6^{\text {th }} \text { iteration } \\
\left|E_{R}\right|=0.00005 \%\end{array}$ & $\begin{array}{c}9.46166121 \\
7^{\text {th }} \text { iteration } \\
\left|E_{R}\right|=0.00306 \%\end{array}$ \\
\hline 02 & 2.25525193 & $\begin{array}{c}2.25525202 \\
3^{\text {rd }} \text { iteration } \\
\left|\mathrm{E}_{\mathrm{R}}\right|=0.0000040 \%\end{array}$ & $\begin{array}{c}2.25525194 \\
3^{\text {rd }} \text { iteration } \\
\left|\mathrm{E}_{\mathrm{R}}\right|=0.0000004 \%\end{array}$ & $\begin{array}{c}2.25525212 \\
3^{\text {rd }} \text { iteration } \\
\left|\mathrm{E}_{\mathrm{R}}\right|= \\
0.0000084 \%\end{array}$ \\
\hline 03 & 0.93697674 & $\begin{array}{c}0.93697840 \\
4^{\text {th }} \text { iteration } \\
\left|\mathrm{E}_{\mathrm{R}}\right|=0.00018 \%\end{array}$ & $\begin{array}{c}0.93697747 \\
4^{\text {th }} \text { iteration } \\
\left|\mathrm{E}_{\mathrm{R}}\right|=0.00008 \%\end{array}$ & $\begin{array}{c}0.93698138 \\
4^{\text {th }} \text { iteration } \\
\left|E_{R}\right|=0.00050 \%\end{array}$ \\
\hline
\end{tabular}




\begin{tabular}{|c|c|c|c|c|}
\hline 04 & 3.20868167 & $\begin{array}{c}3.20870662 \\
6^{\text {th }} \text { iteration } \\
\left|E_{R}\right|=0.00078 \%\end{array}$ & $\begin{array}{c}3.20868230 \\
5^{\text {th }} \text { iteration } \\
\left|E_{R}\right|=0.00002 \%\end{array}$ & $\begin{array}{c}3.20873816 \\
6^{\text {th }} \text { iteration } \\
\left|E_{R}\right|=0.00176 \%\end{array}$ \\
\hline 05 & 0.52495805 & $\begin{array}{c}0.52495910 \\
4^{\text {th }} \text { iteration } \\
\left|\mathrm{E}_{\mathrm{R}}\right|=0.000200 \%\end{array}$ & $\begin{array}{c}0.52495807 \\
4^{\text {th }} \text { iteration } \\
\left|E_{R}\right|=0.000004 \%\end{array}$ & $\begin{array}{c}0.52496060 \\
4^{\text {th }} \text { iteration } \\
\left|E_{R}\right|=0.000486 \%\end{array}$ \\
\hline
\end{tabular}

\section{Conclusions}

From above comparison table we have shown that Milne's (modified) predictor-corrector formulae gives better accuracy and it also can minimize the calculating time as it takes less number of iterations. But, it is yet to implement proposed formulae to the real world problems. Though Milne's (modified) predictor-corrector formula seems to be lengthy process of solving ordinary differential equations, it has following advantages over previous methods. Such as, (i) the previous methods estimates the value of y respecting a given value of $\mathrm{x}$ by means of four initial conditions whereas the Milne's (modified) predictor-corrector formulae estimate the value of $\mathrm{y}$ respecting a given value of $\mathrm{x}$ by means of five initial conditions, which is more logical, (ii) to obtain value of $y$ at any value of $x$, previous methods are need to be calculating up to fourth difference of Newton's formula of forward interpolation but Milne's (modified) predictor-corrector formulae need to be calculating up to fifth difference of Newton's formula of forward interpolation, which will give better accuracy, (iii) at Milne's (modified) corrector formula the co-efficient of $\Delta^{5}$ is zero, then the truncation error converging to zero, this will upgrade the level of accuracy of the method. Next, we are to construct a generalized formula for predictorcorrector method for solving ordinary differential equations of first order and first degree.

\section{Acknowledgement}

Authors are highly grateful to Professor Dr. Munshi Nazrul Islam, Department of Mathematics, University of Chittagong, Bangladesh for his valuable suggestions of this work.

\section{References}

[1] A. RALSTON and P. RABINOWITZ, “A first course in numerical analysis", McGraw-Hill Book Company, Auckland, 1988, PP. 193.

[2] A. R. VASISTHA, V. VASISTHA, "Numerical analysis", KedarNath-Ram Nath, Meerut, 1999, PP.284,288.

[3] B. D. SHARMA, "Differential equations", KedarNath-Ram Nath, Meerut, 2006, PP.06

[4] B. S. GOEL and S. K. MITTAL, "Numerical analysis", Pragati-Prakashan, India, 1995, PP.518

[5] V. N. VEDAMURTHY and N. Ch. S. N. IYENGAR, "Numerical methods", Vikas Publishing house Private Ltd., New Delhi, 2002, PP.11.49, 11.21, 11.58

[6] FRANCIS SCHELD, "Numerical analysis", Schaum's Outline Series, McGraw-Hill, 1988, PP.471.

[7] JAMES B. SCARBOROUGH, “Numerical mathematical analysis” Oxford and IBM Publishing Co. Pvt. Ltd., 1966, PP.354.

[8] S. BALACHANDRA RAO and C. K. SHANTHA, "Numerical methods”, Universities Press India Ltd., Hyderabad, 2000, PP.386.

[9] S. S. SASTRY, 2002. "Introductory methods of numerical analysis", Third edition, Prentic-Hall of India Private Ltd., New Delhi, 2002, PP.303. 